\title{
Improved recovery of higher boiling point volatiles during solvent-assisted flavour evaporation
}

\author{
Rosa C. Sullivan $^{1,2}$ (D) . Colette C. Fagan ${ }^{1}$ (D) . Jane K. Parker ${ }^{1}$ (D)
}

Received: 25 January 2021 / Accepted: 16 June 2021 / Published online: 9 July 2021

(c) The Author(s) 2021

\begin{abstract}
Previously published data show that high levels of fat (50\%) affect the yield of volatile compounds during solvent-assisted flavour evaporation (SAFE). We present new data demonstrating that even low levels of fat $(<10 \%)$ lead to significantly lower yields of high boiling point volatiles during SAFE. Relative recovery during SAFE of a range of volatiles from a cheese extract was measured at varying fat concentrations (1.1-8.7\%) using a single internal standard. Volatiles with higher boiling points had significantly lower relative recoveries, and volatiles were substantially less well recovered from higher fat extracts. When endeavoring to obtain solvent extracts of fatty foods for the purposes of GC-O, it is important to choose the extraction technique which produces solvent extracts closely representing the true composition of the food. We present dilution of solvent extracts prior to SAFE as a potential new approach for high-fat foods which enables high yields of volatiles regardless of boiling point. These data also show that in the absence of C13-labelled standards for quantitation, it is critical to maintain a consistent fat content between samples during SAFE.
\end{abstract}

Keywords Cheese $\cdot$ SAFE $\cdot$ Relative recovery $\cdot$ Fat $\cdot$ Volatiles

\section{Introduction}

Solvent-assisted flavour evaporation (SAFE) is a widely used technique for the removal of fat extracted from foods prior to gas chromatographic analysis. Removal of fat is an essential step in producing solvent extracts from high-fat foods, as the quantity of lipid material is usually significant and, if not removed, causes issues with the concentration of solvent extracts and the contamination of chromatographic equipment. Previous work (Engel et al. 1999; Lewis Jones, personal communication) has indicated that high-fat content affects the relative recovery of volatile compounds during SAFE. Engel et al. (1999) used a 50\% diethyl ether dilution of triacylglycerides as a model organic extract containing a high level of a model fat. Their work demonstrated that SAFE is less effective at recovering volatile compounds when high concentrations of fat are present in the organic

Jane K. Parker

j.k.parker@ reading.ac.uk

1 Department of Food and Nutritional Sciences, University of Reading, Whiteknights, Reading RG6 6DZ, UK

2 Synergy Flavours Ltd, Hillbottom Rd, High Wycombe HP12 4HJ, UK extract. However, Engel et al. (1999) did not investigate the effect of moderate fat levels on the efficacy of SAFE. This topic is of relevance for further investigation as solvent extracts from high-fat foods often contain moderate levels of fat when undergoing SAFE. This is of particular importance in studies focusing on aroma differences between low- and high-fat versions of a food. One such food which has been compared in low- and high-fat form is cheese. The hypothesis of this work was that even moderate to low levels of cheese fat in a solvent extract would affect volatile yields during SAFE.

Cheese aroma is typically studied by extraction of volatile flavour compounds from the cheese matrix, followed by identification and quantification using gas chromatography mass spectrometry (GC-MS). Odorants in cheese can be identified using gas chromatography olfactometry (GC-O) and related techniques such as aroma extract dilution analysis (AEDA). Choice of extraction technique is key to this process since it can have a significant impact on both the quality and quantity of the compounds identified.

Extraction techniques for volatile compounds can be divided into two classes: solvent extraction techniques and headspace techniques. Petersen et al. (2006) previously studied the effect of fat content of cheese on extraction efficiency 
during dynamic headspace extraction. They found significant differences between the recovery of some volatiles from cheeses of varying fat content, which was attributed to the hydrophobicity of the compounds. To facilitate repeat analysis and avoid selectivity based on volatility, solvent extraction is often preferred over headspace extraction techniques.

The focus of this work was on solvent extracts containing cheese fat; however, it is likely that findings will be more widely applicable to extracts from other high-fat foods.

\section{Materials and Methods}

\section{Reagents and Chemicals}

Aroma chemicals and the internal standard solution $(0.500 \%$ 5-methyl-2-hexanone in isopropyl alcohol) were all obtained at $>99 \%$ purity from Synergy (High Wycombe, UK). Diethyl ether was obtained from Sigma-Aldrich Ltd. (Gillingham, UK).

\section{Design of Analyte Mixture and Internal Standard}

To evaluate the efficacy of SAFE across a range of different volatiles, an analyte mixture containing compounds of varying functional group, volatility, and hydrophobicity was designed. In preliminary work, an extract of the cheese without spiked analytes was analysed by GC-MS to confirm that none of the selected analytes was present in the cheese itself, nor were any of the analytes likely to coelute with compound peaks from the cheese. The analyte mixture consisted of each of the compounds displayed in Table 1 (group A) at $0.5 \%$ concentration in isopropyl alcohol. One internal standard (5-methyl-2-hexanone) was added to correct for instrumental drift and minor losses of solvent during SAFE. The internal standard was added to the powdered cheese along with the analyte mixture. It was chosen to have a reasonably low boiling point to maximise recovery during SAFE and make it comparable to the lower boiling point analytes chosen for the study.

\section{Cheese Extract Preparation}

The cheese used during this study was medium cheddar containing 35\% fat, purchased from Tesco (High Wycombe, UK) on the day of analysis and stored at $4{ }^{\circ} \mathrm{C}$ before use. Cheese $(\sim 200 \mathrm{~g})$ was cut into $1 \mathrm{~cm}^{3}$ pieces and frozen rapidly in liquid nitrogen prior to blending in an electric bladebased coffee grinder (Sonifer, Amazon, UK) for $30 \mathrm{~s}$. In triplicate, a portion of cheese $(50 \pm 1 \mathrm{~g})$ was spiked with 200 $\mu \mathrm{L}$ analyte mixture and $200 \mu \mathrm{L}$ internal standard solution (5-methyl-2-hexanone $0.500 \%$ in isopropyl alcohol), left to equilibrate for $5 \mathrm{~min}$ and extracted using $200 \mathrm{ml}$ diethyl ether
Table 1 Boiling points and octanol water partition coefficients $(\log P$ values) of group A (volatiles chosen for analyte mixture) and group B (volatiles used by Engel et al. 1999); data obtained from (a) Scifinder (experimental), (b) ChemSpider (experimental), (c) PubChem (experimental), and (d) ChemSpider (estimated)

\begin{tabular}{llll}
\hline Compounds & Code & $\begin{array}{l}\text { Boiling } \\
\text { points } \\
\left({ }^{\circ} \mathrm{C}\right)\end{array}$ & Log $P$ \\
\hline Group A & & & \\
$\quad$ Ethyl butanoate & EB & $120^{\mathrm{a}}$ & $1.85^{\mathrm{c}}$ \\
Hexanal & HX & $130^{\mathrm{a}}$ & $1.78^{\mathrm{c}}$ \\
2,5-Dimethylpyrazine & DMP & $156^{\mathrm{a}}$ & $0.63^{\mathrm{c}}$ \\
Dimethyl trisulfide & DMTS & $183^{\mathrm{a}}$ & $1.87^{\mathrm{d}}$ \\
Limonene & LM & $176^{\mathrm{a}}$ & $4.57^{\mathrm{c}}$ \\
4-Anisaldehyde & $4 \mathrm{AA}$ & $248^{\mathrm{a}}$ & $1.76^{\mathrm{c}}$ \\
$\gamma$-Decalactone & GDL & $281^{\mathrm{a}}$ & $2.72^{\mathrm{c}}$ \\
Vanillin & VAN & $285^{\mathrm{a}}$ & $1.37^{\mathrm{c}}$ \\
Raspberry ketone & RK & $292^{\mathrm{b}}$ & $0.76^{\mathrm{c}}$ \\
5-Methyl-2-hexanone (internal & & $144^{\mathrm{a}}$ & $1.88^{\mathrm{c}}$ \\
$\quad$ standard) & & & \\
Group B & & & \\
3-Methylbutanoic acid & $3 \mathrm{MBA}$ & $176^{\mathrm{a}}$ & $1.16^{\mathrm{c}}$ \\
Phenylacetaldehyde & PAC & $195^{\mathrm{a}}$ & $1.78^{\mathrm{c}}$ \\
2-Phenylethanol & PEA & $218^{\mathrm{a}}$ & $1.36^{\mathrm{c}}$ \\
(E,E)-2,4-Decadienal & DD & $248^{\mathrm{a}}$ & \\
(E)- $\beta$-Damascenone & BDAM & $274^{\mathrm{a}}$ & $3.41^{\mathrm{b}}$ \\
Vanillin & VAN & $285^{\mathrm{a}}$ & $1.37^{\mathrm{c}}$ \\
3-Hydroxy-4,5-dimethyl-2(5H)- & SOT & $312^{\mathrm{b}}$ & $1.03^{\mathrm{b}}$ \\
$\quad$ & & & \\
\hline
\end{tabular}

by stirring for $1 \mathrm{~h}$. The remaining cheese solids were allowed to settle from the extract and removed by paper filtration and pressed within a filter paper to minimise loss of the extract. A portion of the resulting extract evaporated to dryness confirmed the fat content to be $8.7 \%$. After extraction, four aliquots of $20 \mathrm{ml}$ were separated and diluted respectively to $8.7,4.4,2.2$ and $1.1 \%$ fat. Each extract underwent SAFE and the process was carried out in triplicate. Extracts were analysed by GC-MS before and after SAFE, the pre-SAFE samples containing fat were injected last, as they caused significant dirtying of the chromatographic system.

The analyte mixture and internal standard solution (200 $\mu \mathrm{L}$ each) were also spiked directly into $200 \mathrm{ml}$ diethyl ether producing a " $0 \%$ fat dilution" which underwent SAFE in triplicate and was analysed by GC-MS before and after SAFE.

\section{SAFE Extraction}

Samples underwent SAFE extraction using glassware conforming to that described in previous literature (Engel et al. 1999). The water bath and circulatory water were heated to $40{ }^{\circ} \mathrm{C}$, and the cooled flask was submerged in liquid 
nitrogen. The samples were added dropwise such that consistently low pressure $\left(6-9 \times 10^{-4} \mathrm{kPa}\right)$ was maintained.

\section{GC-MS Analysis of Volatile Compounds}

All volatile analyses were performed on an Agilent 78905977A GC-MS system (Agilent, Stockport, UK) equipped with an autosampler (Agilent, Stockport, UK). Each liquid extract $(3 \mu \mathrm{L})$ was injected in splitless mode onto a DBFFAP polar column $(30 \mathrm{~m}, 0.25 \mathrm{~mm}$ I.D., $0.25 \mathrm{~mm}$ film thickness) (Phenomenex, Macclesfield, UK). The inlet temperature was $240{ }^{\circ} \mathrm{C}$, and the interface temperature was $250{ }^{\circ} \mathrm{C}$. The oven temperature was $45^{\circ} \mathrm{C}$ initially, rising by $4{ }^{\circ} \mathrm{C} / \mathrm{min}$ to $220^{\circ} \mathrm{C}$, and held for $35 \mathrm{~min}$. Helium was used as the carrier gas at $2.2 \mathrm{ml} / \mathrm{min}$. Postcolumn, the signal was split equally between the mass spectrometer, the FPD detector (Agilent, UK, operating in sulfur mode), and the odour port (ODP, Gerstel, UK). The mass spectrometer was operated in electron ionisation mode with a source temperature of $230{ }^{\circ} \mathrm{C}$, a quadrupole temperature of $150{ }^{\circ} \mathrm{C}$, an ionising voltage of $70 \mathrm{eV}$, and a scan range from $\mathrm{m} / \mathrm{z} 40$ to $\mathrm{m} / \mathrm{z} 300$ at $5.3 \mathrm{scans} / \mathrm{s}$. The data were acquired and analysed using Masshunter software (Version 4.5, Agilent, UK). Compounds were identified by first comparing their mass spectra with those contained in the NIST14/Wiley Mass Spectral Database. Identities were confirmed by comparison of their linear retention index against those of authentic standards.

\section{Calculation of Relative Recoveries}

Relative quantitation was performed using the peak areas of the analytes relative to the peak area of an internal standard (5-methyl-2-hexanone) in the same sample. The relative analyte concentrations were calculated using peak areas relative to the internal standard, and the relative recoveries from SAFE were calculated:

$\underline{\text { (Relative concentration in the post-SAFE extract) }} \times 100$

These relative recoveries represent how well a single internal standard behaves when a wide range of compounds are analysed in different matrices.

\section{Statistics}

The relative recovery data for each compound were analysed by one-way analysis of variance (ANOVA) using XLSTAT statistical and data analysis solution (Addinsoft (2020) New York, USA). For those compounds exhibiting significant difference in the ANOVA, Fisher's least significant difference (LSD) test was applied to determine which sample means differed significantly $(p=0.05)$. The Shapiro-Wilk test was used to confirm normality of the data, and the Levene's test to confirm homogeneity of variances. Within 5\% confidence, the data were both normally distributed and had normal variances, suggesting an ANOVA is a valid test for these data.

\section{Results}

Although cheese is composed of various non-volatile components (including proteins, fats and carbohydrates), the non-volatile material extracted into the organic extract of cheese is likely to be largely composed of fat, as fat is readily soluble in diethyl ether. It is unlikely that other more polar components (proteins, carbohydrates) are present above trace levels, so discussion of these results will focus on fat content as the variable influencing yield of volatile compounds.

The results shown in Fig. 1 (see also Table S1) demonstrate that fat content affects the relative recovery of volatile compounds during SAFE. For all compounds except limonene and 2,6-dimethylpyrazine, there was a significant decrease in relative recovery when fat content was increased from 0 to $8.8 \%$. As the boiling point increased, the significant difference was observed when fat content was $\geq 2.2 \%$ (from anisaldehyde onwards) and a significant reduction was observed at $1.1 \%$ fat for the three highest boiling compounds ( $\gamma$-decalactone, vanillin and raspberry ketone). The extent of the reduction was also greatest in the high boiling compounds: at $4.4 \%$ fat, mean relative recovery for $\gamma$-decalactone, vanillin and raspberry ketone were 28,18 , and $3 \%$, respectively. Higher boiling point volatiles and higher concentrations of fat in the extract were both associated with substantially lower relative recoveries during SAFE.

\section{Discussion}

\section{Relative Recovery from Standard in Solvent}

The results for the 0\% fat sample (standard in diethyl ether) agreed closely with previous work (Engel et al. 1999). In both studies, the recoveries from fat-free systems were high, ranging from 80 to $108 \%$ in the present study and 84 to $100 \%$ in the previous work. Neither work suggested that higher boiling point volatiles were less well recovered from the $0 \%$ fat matrix, although this trend was observed when fat was introduced to the matrix. Figure 2 displays the yield data reported previously by Engel et al. (1999) compared to the data from this study in relation to the boiling point of the analytes. While the majority of the analytes differed between the two studies, vanillin was used in both. Previously, the average yield for vanillin in a $0 \%$ fat matrix was reported as $100 \%$ (Engel et al. 1999), which agrees closely with the average relative recovery of $105 \%$ from this study. 


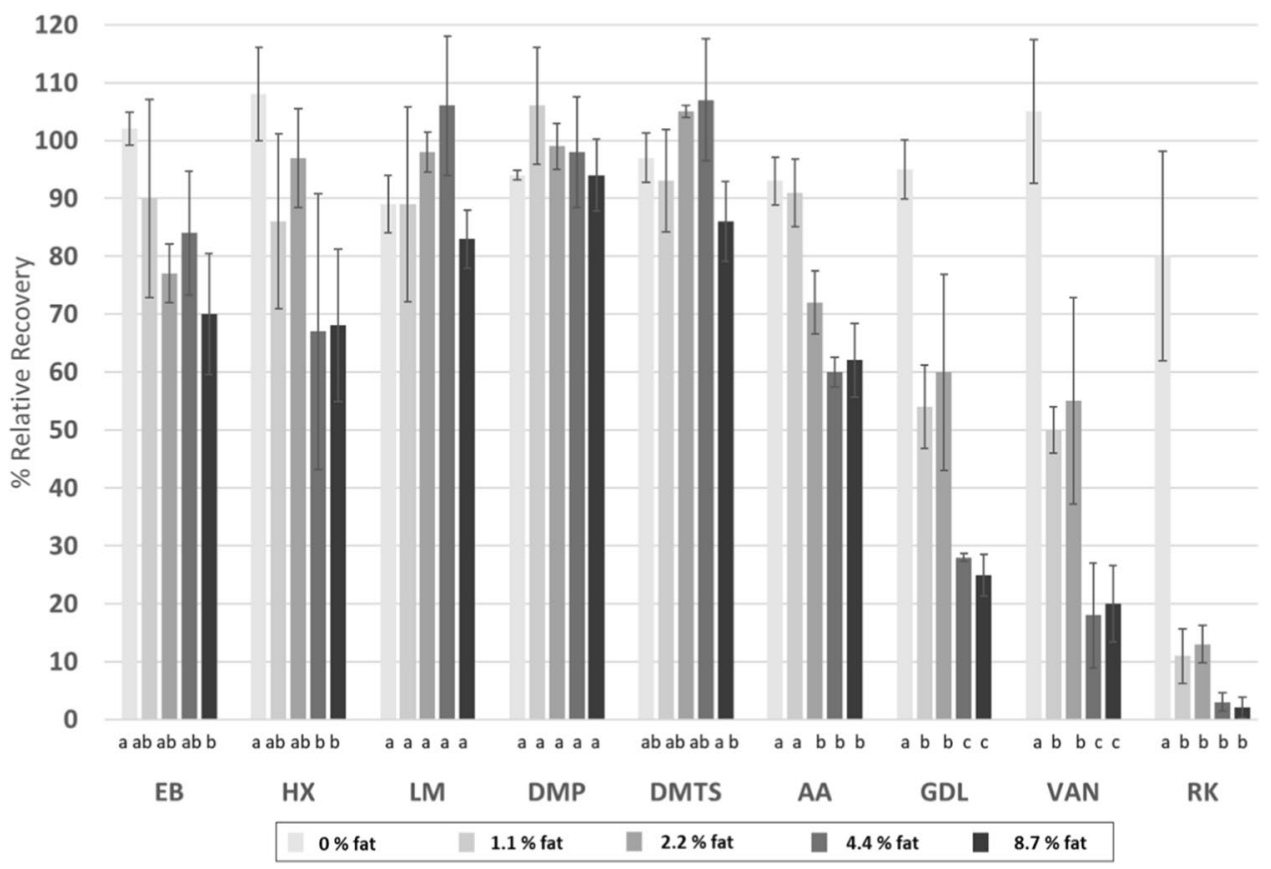

Fig. 1 Bar graph displaying relative recovery data for volatile compounds in solvent extracts of varying fat content during SAFE from 0 to $120 \%$ on the $y$-axis. Compounds are displayed from left to right in order of increasing boiling point and labelled according to abbreviations listed in Table 1. Data shown are mean values from data recorded in triplicate; error bars represent the range for each relative recovery data point. Letters a-c represent significant differences

\section{Comparison to Previous Work on SAFE Yields}

Engel et al. (1999) showed significantly lower yields were obtained from a high-fat (50\%) extract, especially the higher boiling point compounds. Figure 3 shows their yield data

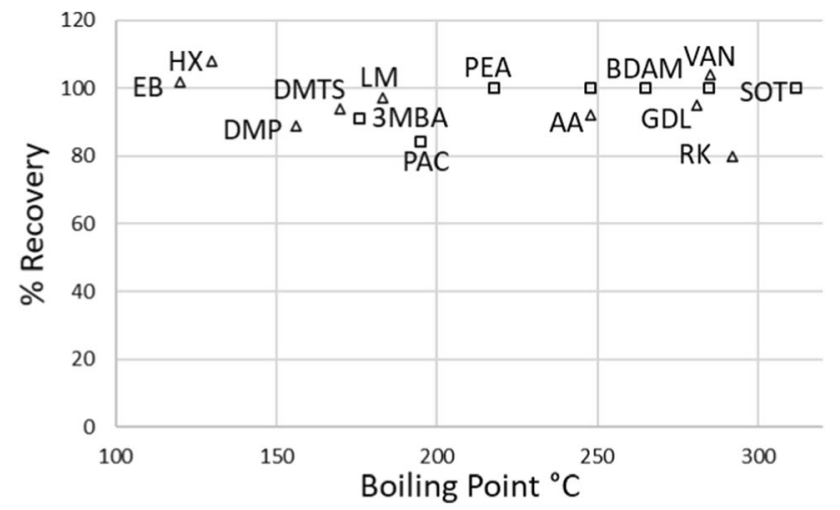

Fig. 2 Relative recovery from solvent extracts containing $0 \%$ fat during SAFE from 0 to $120 \%$ on the $y$-axis, plotted against boiling point of volatile compounds from 100 to $300{ }^{\circ} \mathrm{C}$ on the $x$-axis. Data is a comparison of values presented in previous literature (empty squares) (Engel et al. 1999) and the present work (empty triangles) (see Table 1 for codes). Recoveries are all in the range of 75 to $110 \%$, and no visual trends by boiling point are apparent determined by ANOVA (Fisher's least significant difference (LSD) test, $p=0.05$ ). The bars show recoveries over $80 \%$ in the $0 \%$ fat sample and a general trend for decreased recovery with increased fat content. The difference in recovery between 0 and $1.1 \%$ fat are small and not significant in the most volatile compounds but become significant moving along the $x$-axis to the less volatile compounds

from an extract containing 50\% fat extract. The data show a general trend for lower yield at higher boiling points.

The present study extends the results of Engel et al. (1999) in 50\% fat, to demonstrate that even a moderate

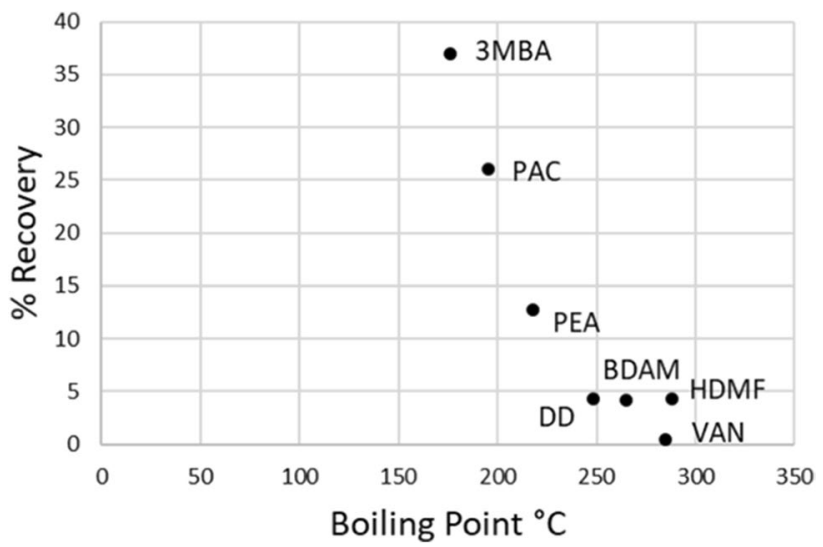

Fig. 3 Relative recovery from solvent extracts containing $50 \%$ fat during SAFE from 0 to $40 \%$ on the $y$-axis, plotted against boiling point of volatile compounds from 100 to $300{ }^{\circ} \mathrm{C}$ on the $x$-axis, data taken from a previous study by Engel et al. (1999) (see Table 1 for codes). The recovery is over $35 \%$ for the lowest boiling point compound (3-methylbutanoic acid), but this decreases with higher boiling points up to $250{ }^{\circ} \mathrm{C}$, after which all recoveries are less than $5 \%$ 
concentration of fat (up to $8.8 \%$ ) in a solvent extract can also significantly affect the yield of volatiles during SAFE. While the previous work used a fat model system composed of a synthetic mixture of triacylglycerides, the present study used real cheese as the matrix. Further studies would be required to determine whether the composition of fat significantly affects the relative recovery during SAFE.

Figure 4 highlights the relationship between boiling point and relative recovery of the volatile during SAFE from the $8.7 \%$ fat extract and shows a similar relationship between relative recovery and boiling point to that observed in the data of Engel et al. (1999). There was no evidence that hydrophobicity was related to the relative recovery during SAFE in the present study, for example, 2,5-dimethylpyrazine and limonene have $\log P$ values of 0.67 and 4.57 , respectively, however, their recoveries from the high-fat cheese extract were very similar. The data suggest that at moderate concentrations of fat, boiling point has a much more significant impact on relative recovery during SAFE than hydrophobicity.

An alternative to SAFE, thin layer high vacuum distillation (TLHVD) was reported by Krings et al. (2003) to demonstrate improved recoveries of volatiles from a highfat (50-90\%) model extract compared to previous relative recovery data for some volatiles using a SAFE method. As with SAFE, high boiling point compounds were less well recovered from the TLHVD system compared to low boiling point compounds, and the authors also found $\log P$ to be related to the relative recovery in a high-fat matrix. They reported a good correlation between the product of the boiling point and $\log P$ values and the recoveries for the range of

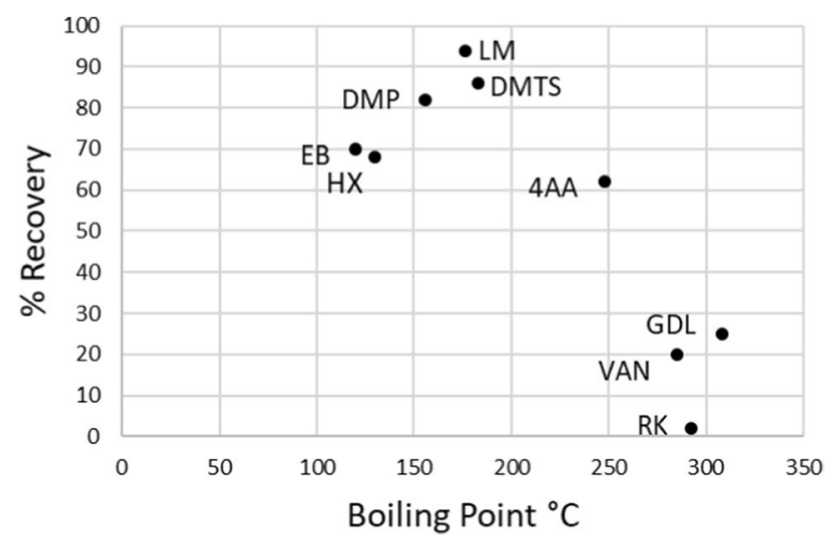

Fig. 4 Relative recovery from solvent extracts containing $8.7 \%$ fat during SAFE from 0 to $100 \%$ on the $y$-axis, plotted against boiling point of volatile compounds from 100 to $300{ }^{\circ} \mathrm{C}$ on the $x$-axis, plotted against boiling point of volatile compounds (see Table 1 for codes). Recoveries for compounds with boiling points between 100 and $150{ }^{\circ} \mathrm{C}$ are around $70 \%$; this initially increases with boiling point to over $90 \%$ recovery for limonene with a boiling point of $176{ }^{\circ} \mathrm{C}$, before decreasing sharply to recoveries between 0 and 30\% for compounds with boiling points over $275^{\circ} \mathrm{C}$ volatile compounds, with the exception of lactones. TLHVD may offer a more effective alternative to SAFE for recovery of volatile compounds from fatty matrices. Further work to is required to determine whether dilution of an extract prior to TLHVD can improve the recovery of high boiling point/ $\log P$ volatiles, especially lactones. Despite promising data on TLHVD, SAFE is a much more widely used technique and is considered the gold standard for isolation of volatiles from fatty matrices. As such, SAFE is the focus of this work and the discussion of the literature to follow.

Recently, SAFE recovery data for a number of compounds from a diethyl ether/dichloromethane (ratio 2:1) bread crumb extract containing a low level of fat (less than $1 \%$ ) were reported (Pico et al. 2018). Given this low level of fat, the recoveries were lower than would be expected when compared to the data from the present study. For example, the recovery of limonene from the bread crumb extract (less than $1 \%$ fat) was $24 \%$, while we report recoveries of 93-106\% from extracts containing $0-8 \%$ fat. The authors reported that matrix effects contributed to low recoveries in their study, however, when they adjusted for the matrix effect for limonene, the calculated extraction efficiency was still only $63 \%$. Furthermore, the data reported did not show a relationship between boiling point and $\%$ recovery.

Though the solvent extract from bread produced by Pico et al. (2018) was comparable to that reported in this study in terms of fat content, the high starch content of bread may have had an impact on the recovery data. Starch has been known to form complexes with volatile compounds (Jeon et al. 2003), especially acids, and this may affect solid-liquid extraction.

\section{Significance to Quantitation and GC-O Studies}

In this study, the aim was to compare the amount of a range of volatiles in a solvent extract pre- and post-SAFE at various fat contents, rather than to accurately quantify their concentrations. A single internal standard was included to correct for any losses of solvent during SAFE. However, low recoveries of high boiling point volatiles relative to a lower boiling point internal standard demonstrate the inaccuracy of using a single internal standard approach for quantifying a broad range of volatiles in post-SAFE extracts. Better techniques for quantitation in these circumstances are well known. For example, using multiple internal standards which represent a broader range of volatiles can improve quantitation somewhat, but chemical differences between the chosen standards and the analytes may still introduce inaccuracies. Standard addition of each of the analytes into the sample at multiple levels to develop a standard addition plot is a better approach to quantitation but requires multiple extractions at different concentrations of spiked analytes. Standard isotope dilution assay (SIDA) is the method of 
choice for quantitation where isotopically labelled standard are available. While dilution of solvent extracts to a low level of fat may significantly improve the recovery of higher boiling point volatiles, other techniques are recommended if accurate quantitation is required.

The results outlined in this study highlight the challenge of using SAFE for obtaining an extract which is representative of high-fat food matrices such as cheese. When comparing extracts by GC-O using techniques such as AEDA, extracts which contained a moderate or high level of fat during SAFE may contain significantly lower quantities of high boiling odorants than the original foodstuff, which may prevent their detection during GC-O. For GC-O comparisons to be effective, it is crucial to obtain solvent extracts which are representative of the aroma of the foodstuff. Quantitation of odorants, even using addition techniques such as stable isotope dilution assay (SIDA), and subsequent recombinant studies may fail to entirely recreate the aroma of foodstuffs in cases where key odorants were not detected during GC-O. Other considerations such as choice of extracting solvent, number of aliquots of solvent used during extraction and extraction methods (e.g. Soxhlet) may all also influence the recovery of volatiles in the extract. To the authors' knowledge, this is the first time a moderate to low-fat content in the extract during SAFE has been reported to significantly affect the yield of volatiles.

\section{Significance to Previously Published Work on Cheese}

Considering the results from the present work, it is possible that the significance of compounds with high boiling points in cheese may have previously been underestimated due to a moderate or high concentration of fat in the solvent extract during SAFE/vacuum distillation extraction. Comparison of the relative recovery of vanillin in the present work and the study of Engel et al. (1999), recorded at $4.4 \%$ and 50\% fat, respectively, indicated approximately a 40 -fold difference, which is significant enough to affect detection by GC-O and FD factors calculated during AEDA. The relative recovery of vanillin was also over twice as high in the $1.1 \%$ extract compared to the $8.7 \%$ extract, indicating that even a difference of low to moderate fat concentration can relative recovery.

Several studies (Carunchia Whetstine et al. 2006; Milo and Reineccius 1997) have used vacuum distillation techniques to determine the key odorants in cheeses containing high levels of fat. Milo and Reineccius (1997) compared FD factors obtained from full fat and $40 \%$ reduced fat cheddar. In this study, an older version of vacuum distillation was used rather than SAFE, but it has been shown to follow similar trends of low recoveries for high boiling point compounds from fatty matrices (Engel et al. 1999).
As such, the vacuum distillation used by Milo and Reineccius (1997) is likely to also have been affected by the fat composition of the solvent extract. Fat contents of the cheeses reported by Milo and Reineccius (1997) were not recorded; however, it is probable that the cheese extracts contained approximately $40 \%$ and $20 \%$ fat respectively due to a difference in the quantities of the two cheeses used. As the key odorants were also quantified by spiking with deuterated standards, the quantitative differences reported between high and low-fat cheese are robust. However, it is possible that there were odorants present in the high-fat cheese which were not detected due to depletion in the high-fat extract during vacuum distillation.

For example, 6-(Z)-dodecen- $\gamma$-lactone reported by Milo and Reineccius (1997) was quantified at a very similar level in both the low- and high-fat cheese, while the low-fat FD factor was 4 times that of the high-fat cheese. This is likely to have been a result of depleted recovery during vacuum distillation of 6-(Z)-dodecen- $\gamma$-lactone from the high-fat cheese extract, due to the high boiling point of this compound. This low recovery would affect the GC-O FD factor but not the quantitation due to the robust quantitation method used.

Drake et al. (2010) also compared FD factors of full and reduced fat cheeses. The extracts of the two cheeses underwent SAFE containing approximately $32 \%$ and $5 \%$ fat, respectively, which is likely to have significantly influenced the volatile recovery of the two extraction procedures, especially affecting volatiles with higher boiling points. Furthermore, compounds were quantified by comparison to an external standard curve obtained by spiking standards into water, rather than the cheese matrices, followed by solvent extraction and SAFE.

In light of the results from the present study, recoveries from the high-fat cheese extract are likely to differ significantly from those used to generate the external standard curve and from the low-fat cheese extract. Further investigation into the relative recovery of volatiles from the two cheese matrices may be required to confirm their findings.

A key finding of the work of Drake et al. (2010) was increased burnt sugar notes in the 9-month aged low-fat cheese compared to the 9-month aged high-fat cheese. The authors attributed the burnt note in the low-fat cheese to furanone compounds; furaneol, homofuraneol and sotolone, for which higher FD factors were obtained for the low-fat cheese extract than for the high-fat cheese extract. With the exception of homofuraneol, the differences in FD factors between low and high-fat cheese were very large. For example, the FD factor of sotolone was $<3$ in 9-month-aged highfat cheese, but 531,441 in the 9-month-aged low-fat cheese. However, when sotolone was quantified the differences in concentration between low- and high-fat cheese were shown to be less than a single order of magnitude. 
In light of the results from the present study, the high-fat cheese extract obtained by Drake et al. (2010) is likely to have been significantly depleted of higher boiling point compounds during SAFE. This may have led to artificially low FD factors for furanones in the high-fat cheese and also have affected the external standard quantitation. As the difference in FD factors between the low- and high-fat cheese were so large, the conclusion that furanones contribute to burnt notes in low-fat cheese is likely to be robust. However, depletion of the high boiling point compounds in the high-fat sample may explain the poor correlation between FD factors and concentration.

These authors also discussed lactones as key contributors to milk-fat flavour; however, the FD factors for most lactones were similar in the low- and high-fat cheeses despite a significantly lower milk-fat score in the sensory study for the low-fat cheese. This sensory difference is a logical result as lactones are derived from triglycerides precursors which might be present in lower amounts in the lower-fat cheese. The results of the present study raise the possibility that the recoveries of lactones may have been significantly reduced in the extract from the high-fat cheese, leading to artificially low FD factors.

\section{Recommendations}

To ensure solvent extracts for GC-O studies are closely representative of foods, we demonstrate that dilution of fatty solvent extracts prior to SAFE significantly improves yields of high boiling point volatiles. Dilution prior to SAFE would make a sensible addition in studies comparing the key odorants between low and high-fat versions of the same food, such as cheese. Likewise, it has been shown that multiple aliquots of extraction solvent can be used to increase recoveries when extracting volatiles from foods, this approach could also serve a dual purpose of diluting the fat content in the extract prior to SAFE.

Quantitation from fatty matrices during SAFE is best performed by including multiple, appropriately selected, internal standards, ideally C13-labelled analogues of the analytes of interest. However, in the absence of C13-labelled standards, dilution of the extract prior to SAFE may increase recovery of higher boiling point volatiles relative to a single internal standard.

\section{Conclusion}

This study has demonstrated that even low concentrations of fat in the solvent extract can have a significant impact on yields of volatile compounds during SAFE. Higher levels of fat in the solvent extract and higher boiling points of the analytes were both associated with lower relative recoveries during SAFE. Dilution of cheese extracts to a low level of fat lead to better relative recoveries of high boiling point volatiles. This approach could enable more accurate comparison of volatile compounds in cheeses of differing fat content. It could also ensure that solvent extracts of high fat foods, such as cheese, are representative in their aroma for the purposes of GC-O studies. Given the recent focus on the production of fat-reduced alternatives to high-fat foods, these findings are important for comparison of aroma profiles in standard and reduced-fat products.

Supplementary Information The online version contains supplementary material available at https://doi.org/10.1007/s12161-021-02074-5.

Acknowledgements RS thanks Synergy Flavours Ltd., High Wycombe for financial and technical support for her doctoral studies.

Author Contribution All authors contributed to the study conception and design. Material preparation, data collection, and analysis were performed by RS. The first draft of the manuscript was written by RS, and all authors commented on subsequent versions of the manuscript. All authors read and approved the final manuscript.

Funding Funding was provided by Synergy Flavours to support RS in her doctoral studies.

Data Availability Not applicable.

Code Availability Not applicable.

\section{Declarations}

Ethical Approval This article does not contain any studies with human participants or animals performed by any of the authors.

Informed Consent Not applicable.

Conflict of Interest Rosa Sullivan declares that she has no conflict of interest. Colette Fagan declares that she has no conflict of interest. Jane Parker declares that she has no conflict of interest.

Open Access This article is licensed under a Creative Commons Attribution 4.0 International License, which permits use, sharing, adaptation, distribution and reproduction in any medium or format, as long as you give appropriate credit to the original author(s) and the source, provide a link to the Creative Commons licence, and indicate if changes were made. The images or other third party material in this article are included in the article's Creative Commons licence, unless indicated otherwise in a credit line to the material. If material is not included in the article's Creative Commons licence and your intended use is not permitted by statutory regulation or exceeds the permitted use, you will need to obtain permission directly from the copyright holder. To view a copy of this licence, visit http://creativecommons.org/licenses/by/4.0/.

\section{References}

Carunchia Whetstine ME, Drake MA, Nelson BK, Barbano DM (2006) Flavor profiles of full-fat and reduced-fat cheese and cheese fat made from aged cheddar with the fat removed using a novel process. J Dairy Sci 89(2):505-517. https://doi.org/10.3168/jds. S0022-0302(06)72113-0 
Drake MA, Miracle RE, McMahon DJ (2010) Impact of fat reduction on flavor and flavor chemistry of Cheddar cheeses. J Dairy Sci 93(11):5069-5081. https://doi.org/10.3168/jds.2010-3346

Engel W, Bahr W, Schieberle P (1999) Solvent assisted flavour evaporation - a new and versatile technique for the careful and direct isolation of aroma compounds from complex food matrices. Eur Food Res Technol 209:237-241. https://doi.org/10.1007/s0021 70050486

Jeon Y-J, Vasanthan T, Temelli F, Song B-K (2003) The suitability of barley and corn starches in their native and chemically modified forms for volatile meat flavor encapsulation. Food Research International 36:349-355. https://doi.org/10.1016/S0963-9969(02) 00226-0

Krings U, Banavara DS, Berger RG (2003) Thin layer high vacuum distillation to isolate the flavor of high-fat food. Eur Food Res Technol 217:70-73. https://doi.org/10.1007/s00217-003-0700-9

Milo C, Reineccius GA (1997) Identification and quantification of potent odorants in regular-fat and low-fat mild cheddar cheese.
J Agric Food Chem 45(9):3590-3594. https://doi.org/10.1021/ jf970152m

Petersen MA, Tammam AA, Ardö Y (2006) Spiking as a method for quantification of aroma compounds in semi-hard cheeses. In: W.L.P Bredie \& M.A. Petersen (Eds.), Flavour Science: Recent Advances and Trends. Developments in Food Science Vol 43:221224 https://doi.org/10.1016/S0167-4501(06)80053-1

Pico J, Oduber F, Gómez M, Bernal J (2018) Analytical feasibility of a solvent-assisted flavour evaporation method for aroma analyses in bread crumb. J Sep Sci 41:3902-3909. https://doi.org/10.1002/ jssc. 201800336

Publisher's Note Springer Nature remains neutral with regard to jurisdictional claims in published maps and institutional affiliations. 